\title{
On the structure of quasiconvex hulls
}

\author{
by \\ Kewei ZHANG \\ Department of Mathematics, Heriot-Watt University, Riccarton, \\ Edinburgh EHI4 4AS UK. \\ kewei@ma.hw.ac.uk \\ Current Address: Department of Mathematics, Macguarie University, \\ Sydney NSW 2109 Australia. \\ kewei@mpce.mg.edu.au
}

\begin{abstract}
We define the set $K_{q, e} \subset K$ of quasiconvex extreme points for compact sets $K \subset M^{N \times n}$ and study its properties. We show that $K_{q, e}$ is the smallest generator of $Q(K)$-the quasiconvex hull of $K$, in the sense that $Q\left(K_{q, \dot{e}}\right)=Q(K)$, and that for every compact subset $W \subset Q(K)$ with $Q(W)=Q(K), K_{q, e} \subset W$. The set of quasiconvex extreme points relies on $K$ only in the sense that $Q(K)_{q, e} \subset K_{q, e} \subset \overline{\left[Q(K)_{q, e}\right]}$. We also establish that $K_{e} \subset K_{q, e}$, where $K_{e}$ is the set of extreme points of $C(K)$-the convex hull of $K$. We give various examples to show that $K_{q, e}$ is not necessarily closed even when $Q(K)$ is not convex; and that for some nonconvex $Q(K)$, $K_{q, e}=K_{e}$. We apply the results to the two well and three well problems studied in martensitic phase transitions.

(C) 1998 L'Association Publications de l'Institut Henri Poincaré. Published by Elsevier B.V. All rights reserved
\end{abstract}

\section{INTRODUCTION}

A basic property of compact convex sets in $\mathbb{R}^{n}$ is that they are the closed convex hulls of their extreme points (Krein-Milman Theorem [Ru]). Suppose $K \subset \mathbb{R}^{n}$ is compact and convex, then $K=C\left(K_{e}\right)$ where $K_{e}$ is the set of its extreme points. Also, for every compact $W \subset K$ such that $C(W)=K$, we have $K_{e} \subset W$. We may say that $K_{e}$ is the smallest generator of the convex set $K$. In this paper we introduce a similar notion for quasiconvex sets studied in the variational approach to material phase 
transitions, and we show that the set $K_{q, e}$ of quasiconvex extreme points is the smallest generator of the quasiconvex set $K$. It turns out that $K_{q, \text { e }}$ is a natural generalization of $K_{\epsilon}$. For $K \subset M^{1, n} \cong \mathbb{R}^{n}, K_{q, e}=K_{e}$ (see Example 4.1).

For a compact subset $K \subset M^{N \times n}$, we may define its quasiconvex hull $Q(K)$ by cosets of quasiconvex functions [Sv1], or alternatively, by direct quasiconvex relaxations of the distance functions to the set [Z2]. In fact, the study of properties like quasiconvex hulls for sets in $M^{N \times n}$ goes back to J. M. Ball [B13]. A compact subset $K \subset M^{N \times n}$ is called quasiconvex if $Q(K)=K$ (for more precise definitions, see $\S 2$ below). Examples of quasiconvex sets include the level sets of quasiconvex functions, that is $K=\left\{P \in M^{N \times n}, f(P) \leq \alpha\right\}$ where $\alpha \in \mathbb{R}$ and $f: M^{N \times n} \rightarrow \mathbb{R}$ quasiconvex.

Let $\left(u_{j}\right)$ be any bounded sequence in $W^{1, \infty}\left(\Omega, \mathbb{R}^{N}\right)$, approaching a compact set $K \subset M^{N \times n}$ in the sense that $\operatorname{dist}\left(D u_{j}(x), K\right) \rightarrow 0$ almost everywhere as $j \rightarrow \infty$. We further assume that $u_{j} \stackrel{*}{\rightarrow} u$ in $W^{1, \infty}\left(\Omega, \mathbb{R}^{N}\right)$ in the weak-* sense (from now on, $\stackrel{*}{\rightarrow}$ denotes weak-* convergence). Heuristically, the quasiconvex hull $Q(K)$ of $K$ is the smallest closed set $Q(K)$ such that $D u(x) \in Q(K)$ almost everywhere. This means $Q(K)$ is closed under weak-* convergence. A compact set $K$ is quasiconvex if it is weak-* closed.

Let us use the level set mentioned above as an example, that is,

$$
K=\left\{P \in M^{N \times n}, f(P) \leq \alpha\right\}
$$

where $\alpha \in \mathbb{R}$ and $f: M^{N \times n} \rightarrow \mathbb{R}$ quasiconvex. We assume that $K$ is compact. Let $u_{j} \stackrel{*}{\rightarrow} u$ in $W^{1, \infty}\left(\Omega, \mathbb{R}^{N}\right)$ and $\operatorname{dist}\left(D u_{j}(x), K\right) \rightarrow 0$ almost everywhere as $j \rightarrow 0$. Let $\left\{\nu_{x}\right\}_{x \in \Omega}$ be the family of Young measures corresponding to $\left(D u_{j}\right)$ (see $[\mathrm{T}, \mathrm{B} 12]$ and Lemma 2.6 bellow). Then we have $\operatorname{supp} \nu_{x} \subset K^{\prime}$ and

$$
\int_{K} f(\lambda) d \nu_{x}(\lambda) \geq f(D u(x))
$$

(see, for example [BZ, KP]) for almost every $x \in \Omega$. Here we have used the fact that $f$ is quasiconvex to obtain the inequality above. Now, from the definition of $K$, the left hand side of the above inequality is less than $\alpha$, hence $f(D u(x)) \leq \alpha$, which gives $D u(x) \in K$ almost everywhere. $K$ is a quasiconvex set.

The study of quasiconvex hulls and quasiconvex sets is motivated by the variational approach to martensitic phase transitions and material 
microstructures [ $\mathrm{BJ} 1, \mathrm{BJ} 2, \mathrm{CK}, \mathrm{K}, \mathrm{Sv} 2, \mathrm{MS}$ ]. A natural question in the study of quasiconvex sets or quasiconvex hulls of a given set is to ask whether there exists a 'smallest' subset $K_{0}$ of a quasiconvex set $K$ such that $Q\left(K_{0}\right)=K$. In this paper we show that the following set turns out to be the smallest generator of the quasiconvex set $K$.

Definition 1.1. - Let $K \subset M^{N \times n}$ be non-empty and compact.

$P \in K$ is called a quasiconvex extreme point of $K$ if for every gradient homogeneous Young measure $\nu$ supported in $K$ with $\int_{K} \lambda d \nu(\lambda)=P$, then $\nu=\delta_{P}$ - the Dirac mass supported at $P$.

The set of all quasiconvex extreme points of $K$ is denoted by $K_{q, e}$.

Gradient homogeneous Young measures are generated by bounded $W^{1, \infty}$ sequences, while a minimizing sequence of the functional $\int_{\Omega} \operatorname{dist}^{p}\left(D u_{j}, K\right) d x \rightarrow 0$ is appearently only bounded in $W^{1, p}$. However, a result in [Z1] shows that if the a family of gradient Young measures has uniformly bounded supports, we can find a bounded sequence in $W^{1, \infty}$ which generate the same family of gradient Young measures. Intuitively, the gradient Young measure represents the oscillation of a weakly (weak-*) convergent sequence while the quasiconvex hull of a set consists of all possible averages of the Young measures supported in the set [B13, BJ1, $\mathrm{BJ} 2, \mathrm{KP}, \mathrm{BFJK}]$. Quasiconvex extreme points are those which can only be represented by themselves, that is, by Dirac masses. We may naturally guess that they are the building bricks for a quasiconvex set. Theorem 1.1 justifies this. The definition of quasicovex extreme points by using gradient homogeneous Young measures is natural. As pointed out by J. Kristensen to the author, that in an abstract setting for convex sets, Choquet points and the Choquet boundary are defined by using positive measures [A].

We show in Theorem 1.2 below that $K_{q, e}$ is not empty whenever $K \neq \emptyset$. In fact, we show that $K_{q, e} \supset K_{e}$.

This paper answers the question what the smallest generator of a quasiconvex set is. We do not intend, in this paper, to answer the question of how the quasiconvex set is generated by its smallest generator. This second question seems to be a rather deep one (see [BFJK, MS]).

THEOREM 1.1. - Suppose $K \subset M^{N \times n}$ is compact and quasiconvex. Then $K_{q, e}$ is the smallest generator of $K$ in the sense that

(i) $K_{q, e}$ is a generator of $K, Q\left(K_{q, e}\right)=K$.

(ii) $K_{q, e}$ is the smallest generator of $K$, that is, if $W \subset K$ is compact and $Q(W)=K$, then $K_{q, e} \subset W$. 
COROLlaRY 1.1. - Suppose $K \subset M^{N \times n}$ is compact and quasiconvex, and $V \subset K, W \subset K$ are two compact generators of $K, Q(V)-Q(W)-K$. Then $V \cap W$ is also a compact generator of $K$.

Corollary 1.1 is a direct consequence of Theorem 1.1. However, I do not know whether it can be proved directly without applying Theorem 1.1.

Remark 1.1. - Theorem 1.1 provides a 'minimal' representation of points in a quasiconvex set by the closure of the set of its quasiconvex extreme points via gradient homogeneous Young measures. More precisely, we have that for every $P \in K$, there exists a gradient homogeneous Young measure $\nu$ supported in $\overline{\left[K_{q, \mathrm{e}}\right]}$ such that

$$
P=\int \frac{}{\left[K_{q, \varepsilon}\right]} \lambda d \nu(\lambda)
$$

For more details, see Lemma 3.1 below. Notice that $K_{q, e}$ is not necessarily closed (see Examples 4.1, 4.2).

The following result gives the relation between $K_{q, e}$ and $K_{e}$ for a compact set $K \subset M^{N \times n}$. Notice that in convex analysis, it is well known that $K_{e}=[C(K)]_{e} \subset K$ for any compact set $K \subset \mathbb{R}^{n}$ [Ro].

THEOREM 1.2. - Suppose $K \subset M^{N \times n}$ is non-empty and compact. Then $K_{q, e}$ is not empty. In fact, $K_{\epsilon} \subset K_{q, e}$. Therefore $C\left(K_{q, e}\right)=C(K)$, where $K_{e}$ is the set of extreme points of $C(K)$.

The following result shows that the set of quasiconvex extreme points $K_{q, e}$ of a compact set $K$ depends only on the set, not on the quasiconvex hull $Q(K)$. This indicates that the quasiconvex extreme point is an intrinsic property of the compact set $K$. At present, very few examples are known of explicit forms of $Q(K)$ for a given set $K$.

THEOREM 1.3. - Suppose $K \subset M^{N \times n}$ is non-empty and compact. Then

$$
Q(K)_{q, e} \subset K_{q, e} \subset \overline{\left[Q(K)_{q, e}\right]} .
$$

Notice that for a bounded set $W \subset M^{N \times n}, Q(W)=Q(\bar{W})$ and $Q(W)$ is always compact whenever $W$ is bounded (consult the definition of quasiconvex hulls in $\S 2$ ).

THEOREM 1.4. - Suppose $K$ is compact and $C(K)$ does not have rank-one connections. Then $K_{4, \text { e }}=K=Q(K)$.

In the following result, we use the supporting chains (see Definition 2.9) to describe the structure of $K_{q, e}$ for a compact convex set $K$. 
THEOREM 1.5. - Suppose $K$ is compact, convex with more than one element, and $K$ has rank-one connections. Then

$$
K_{q, e}=\bigcup_{\mathcal{E}}\left(E_{\mathcal{E}} \cap K\right)
$$

where the union is taken over all of the end planes of supporting chains $\{\mathcal{E}\}$ of $K$. In particular, when $K$ is strictly convex, $K_{q, e}=K_{e}$.

We also give some examples to show that (1): $K_{q, e}$ can be non-compact; (2) we can have $K_{q, e}=K_{e}$ even when $Q(K)$ is not convex. We then apply our results on quasiconvex extreme points to the two-well and three-well problems in martensitic phase transitions. For the two-well case, that is, when $K=S O(n) \cup S O(n) H$, we have $K_{q, e}=K$. For the three well case, we prove that $K_{q, e}$ is either $K$ itself or a subset of two wells. We then make some remarks for the general case.

Remark 1.2. - Since we may define other 'semiconvex' hulls, such as rank-one convex hulls, polyconvex hulls the same fashion as quasiconvex hulls (see [Sv2]), it is natural to ask whether we can use subclasses of probability measures to define corresponding extreme points. An interesting question then is whether for a given compact set, rank-one extreme points are the same as quasiconvex extreme points. We do not intend to answer this questions here. However, I guess that the answer is negative. An example might be constructed by using the famous counterexample that rank-one convexity does not implies quasiconvexily due to Šverák [Sv3].

In order to prove the results mentioned above, we use the properties of gradient homogeneous Young measures generated by gradients (see [KP]), the results of quasiconvex hulls, and quasiconvex functions and the basic theory of convex analysis. In $\S 2$, some preliminary results are given. In $\S 3$, we first establish a lemma (Lemma 3.1) to connect homogeneous Young measures and quasiconvex hulls. Then we prove our results listed above. Some explicit examples of $K_{q, c}$ are exhibited in $\S 4$. We apply our results to the two-well and three-well problems in $\S 5$.

\section{PRELIMINARY RESULTS}

We denote by $M^{N \times n}$ the space of all real $N \times n$ matrices with $\mathbb{R}^{N n}$ norm. If $E \subset M^{N \times n}$ is a linear subspace, we write $P_{E}$ and $P_{E^{-}}$as the orthogonal projections from $M^{N \times n}$ to $E$ and its orthogonal complement 
$E^{\perp}$ respectively. meas $(U)$ is the Lebesgue measure of a measurable subset $U \subset \mathbb{R}^{n}$ and

$$
\operatorname{dist}(Q, K)=\inf _{P \in K^{-}}|Q-P|
$$

denotes the distance function from a point $Q \in M^{N \times n}$ to a set $K \subset M^{N \times n}$. From now on $\Omega$ denotes a non-empty, open and bounded subset of $\mathbb{R}^{n}$. For a given set $K \subset \mathbb{R}^{s}$, int $K, \bar{K}$ and $\partial K$ denote its interior, closure and boundary. We denote by $D u$ the gradient of a (vector-valued) function $u$ and we define the space $C_{0}^{k}\left(\Omega, \mathbb{R}^{N}\right)$, the $L^{p}$ spaces and Sobolev spaces $W^{1, p}$ in the usual way. We say that $K \subset M^{N \times n}$ has a rank-one connection if there exist $A, B \in K$ such that $\operatorname{rank}(A-B)=1$. The support of a measure $\nu$ is denoted by $\operatorname{supp} \nu$.

Let $f: M^{N \times n} \rightarrow \mathbb{R}$ be a continuous function. $f$ is quasiconvex (c.f. [B11,Mo,D]) in $M^{N \times n}$ if for every open and bounded subset $\Omega$ of $\mathbb{R}^{n}$, every $P \in M^{N \times n}$ and every $\phi \in C_{0}^{\infty}\left(\Omega, \mathbb{R}^{N}\right)$,

$$
\int_{\Omega} f(P+D \phi(x)) d x \geq \int_{\Omega} f(P) d x
$$

The class of quasiconvex functions is independent of the choice of $\Omega$. It is well known now that $I(u)=\int_{\Omega} f(D u) d x$ is lower semicontinuous in the Sobolev space $W^{1, \infty}\left(\Omega, \mathbb{R}^{N}\right)$, in the weak-* sense if and only if $f$ is quasiconvex (see [Mo, Bll, AF]). Suppose in addition, $f$ satisfies $0 \leq f(P) \leq C\left(1+|P|^{p}\right)$ for $P \in M^{N \times n}$, for some constants $C>0$ and $p \geq 1$, then $I(\cdot)$ is weakly lower semicontinuous in $W^{1, p}\left(\Omega, \mathbb{R}^{N}\right)$ if and only if $f$ is quasiconvex (see $[\mathrm{AF}]$ for the general statements and proofs).

For a given function, we can consider its quasi-convexification (quasiconvex relaxation):

Definition 2.1. - ([D]). Suppose $f: M^{N \times n} \rightarrow \mathbb{R}$ is continuous. The quasiconvexification of $f$ is defined by

$$
\sup \{g \leq f ; g \text { quasiconvex }\}
$$

and will be denoted by $Q f$.

Proposition 2.2. - (see [D]). Suppose $f: M^{N \times n} \rightarrow \mathbb{R}$ is continuous, then

$$
Q f(\mu)=\inf _{\phi \in C_{0}^{\infty}\left(\Omega ; \mathbb{F}^{N}\right)} \frac{1}{\operatorname{meas}(\Omega)} \int_{\Omega} f(P+D \phi(x)) d x,
$$

where $\Omega \subset \mathbb{R}^{n}$ is a bounded domain. $Q f$ is quasiconvex. In particular the infimum in (2.1) is independent of the choice of $\Omega$. 
In the variational approach to martensitic phase transitions, the integrand $f$ is sometimes in a special form where $f \geq 0$, and $f(P)=0$ if and only if $P \in K$, where $K \subset M^{N \times n}$ is a compact set (see [BJ1, BJ2, K, BFJK, $\mathrm{CK}, \mathrm{K}]$ ). Such functions are in general not quasiconvex. Suppose $\left(u_{j}\right)$ is a bounded sequence in the Sobolev space $W^{1, p}\left(\Omega, \mathbb{R}^{N}\right)$, such that $I\left(u_{j}\right) \rightarrow 0$, as $j \rightarrow \infty$, we are interested in the oscillating behaviour of the sequence $\left(D u_{j}\right)$ and the possible 'microstructures' it may generate. The following concept of quasiconvex hull for a set $K \subset M^{N \times n}$ is naturally introduced.

Definition 2.3. - ([Sv2]). For a subset $K$ of $M^{N \times n}$, the quasiconvex hull $Q^{(s)}(K)$ of $K$ is defined by

$$
\begin{aligned}
& Q^{(s)}(K)=\left\{X \in M^{N \times n}, f(X) \leq \sup _{Y \in K} f(Y)\right. \\
& \left.\quad \text { for every quasiconvex } f: M^{N \times n} \rightarrow \mathbb{R}\right\} .
\end{aligned}
$$

Clearly, if $K$ is bounded,

$$
\bar{K} \subset Q^{(s)}(K)=Q^{(s)}(\bar{K}) \subset C(\bar{K}),
$$

$C(\bar{K})$ being the convex hull of $\bar{K}$.

In [Sv2], the above definition of quasiconvex hull is given for all $K \subset M^{N \times n}$. For a compact set $K, Q^{(s)}(K)$ is independent of the choice of the growth of quasiconvex functions in the definition (see Proposition 2.5 below). However, for unbounded $K$, if we restrict the choice of quasiconvex functions to satisfy a particular growth condition at infinity, then the quasiconvex hulls thus defined may depend on the growth rate of the quasiconvex functions $[Y, Z 2, Z 3]$. Therefore we define the quasiconvex hull here in a different way. However, they are equal when $K$ is compact.

DeFINITION 2.4. - ([Z2]). Let $K \subset M^{N \times n}$. Then the p-quasiconvex hull of $K$ for $1 \leq p<\infty$ is defined by

$$
Q_{p}(K)=\left\{A \in M^{N \times n}, \operatorname{dist}^{p}(A, K)=0\right\},
$$

$Q \operatorname{dist}^{p}(\cdot, K)$ being the quasiconvexification of the p-distance function $\operatorname{dist}^{p}(\cdot, K)$ to $K$.

It is easy to see that $Q_{p}(K)=Q_{p}(\bar{K})$, and $Q_{p}(K)$ is closed. For a compact set $K \subset M^{N \times n}$, we have

Proposition 2.5. - (see [Z2] and the Appendix of this paper). Suppose $K \subset M^{N \times n}$ is compact. Then

$$
Q^{(s)}(K)=Q_{p}(K)
$$

for all $p \in[1, \infty)$.

Vol. 15. $n^{\circ}$ 6-1998. 
Because of this result, we may define the quasiconvex hull of $K$ as $Q(K)=Q^{(s)}(K)=Q_{p}(K)$ for all $p \in[1, \infty)$ when $K$ is compact. The advantage of Definition 2.4 is that to study the quasiconvex hull of a compact set $K$, we need only to deal with one particular function, say $Q \operatorname{dist}^{2}(\cdot, K)$ or $Q \operatorname{dist}(\cdot, K)$. We will show the advantage of this quantitative description of quasiconvex hulls in the proofs of Theorem 1.1, 5.1 and 5.2.

Lemma 2.6. (Young measures). - (see, for example [T, B12]). Suppose $\left(U_{k}\right)$ is a bounded sequence in $L^{\infty}\left(\Omega ; R^{s}\right)$, and for some compact set $K \subset R^{s}$, meas $\left(\left\{x \in \Omega: U_{k}(x) \notin G\right\}\right) \rightarrow 0$ as $k \rightarrow \infty$ for every open set $G \supset K$. Then there exists a subsequence (still denoted by $U_{k}$ ) and an associated family of probability measures $\nu_{x}$ on $R^{s}$ such that (i) $\nu_{x}$ is supported on $K$ for almost every $x \in \Omega$ : (ii) for any continuous function $\psi$ on $R^{*}, \psi\left(U_{k}\right)$ converges in the weak-* sense to the function $x \rightarrow \int_{R^{*}} \psi(\lambda) d \nu_{x}(\lambda)$.

In the above definition, if the sequence $U_{k}$, has the form $U_{k}-D u_{k}$, where $\Omega \subset \mathbb{R}^{n}$ is open and bounded, and $\left(u_{k}\right)$ is a bounded sequence in $W^{1, \infty}\left(\Omega, \mathbb{R}^{s}\right)$, then the corresponding Young measure $\nu_{. r}$ is called Young measure limit of gradients or gradient Young measure (see [KP, BFJK]). The Young measure is trivial if $\nu_{x}$ is a Dirac measure for a.e. $x$. In this case there exists a function $u$ such that $\nu_{x}$ is the Dirac measure at $D u(x)$, and up to a subsequence, $D u_{k} \rightarrow D u$ almost everywhere. In general, the Young measure may be nontrivial.

One of the restrictions of Young measure limit of gradients is that for every quasiconvex function $f: M^{N \times n} \rightarrow \mathbb{R}$,

$$
\int_{\mathrm{supp} \nu_{x}} f(\lambda) d \nu_{: r} \geq f\left(\int_{\mathrm{supp} \nu_{s}} \lambda d \nu_{x:}\right)
$$

for almost every $x \in \Omega$ (see for example, [BZ, KP, BFJK]).

LEMMA 2.7. (gradient homogeneous Young measures). (see [KP] for a more general statement). Let $\left\{\nu_{x}\right\}_{x \in \Omega}$ be a family of gradient Young measures $\left\{\nu_{x}\right\}_{x \in \Omega}$ with bounded supports, that is $\operatorname{supp} \nu_{x} \subset K$, where $K$ is a compact subset of $M^{N \times n}$ and $\int_{K} \lambda d \nu_{x_{0}}(\lambda)=P_{0}$ for almost every $x_{0} \in \Omega$. Then for almost every $x_{0} \in \Omega$, there exists a bounded sequence $\left(\phi_{k}\right)$ in $W_{0}^{1, \infty}\left(D, \mathbb{R}^{N}\right)$ such that the corresponding family of gradient Young measures $\left\{\bar{\nu}_{y}\right\}$ of the squence $\left(P_{0}+D \phi_{k}\right)$ satisfy $\bar{\nu}_{y}=\nu_{x_{0}}$ for almost every $y \in D$, where $D$ is the unit open hypercube in $\mathbb{R}^{n} . \bar{\nu}_{y}$ is called a gradient homogeneous Young measure.

From now on, we mean by homogeneous Young measures as gradient homogeneous Young measure (we write HYM for simplicity) by $\nu$ and write its integral average on its compact support $K$ as $\bar{\nu}_{K^{-}}:=\int_{K} \lambda d \nu(\lambda)$. 
The following is a collection of facts from finite dimensional convex analysis (cf. [Ro, Th 2.4, 6.3, 11.5, 11.6 18.8]),

Proposition 2.8. - Suppose $K \subset \mathbb{R}^{n}$ is a compact convex set with more than one element. Then

(i) $K$ has a dimension $m \leq n,(m \geq 1)$ and $K$ is contained in a m-dimensional plane $E_{m} \subset \mathbb{R}^{n}$;

(ii) with the subspace topology induced from $\mathbb{R}^{n}$ on $E_{m}, K$ is the closure of its interior points int $K$, that is, $K=\overline{\operatorname{int} K}$;

(iii) for every convex subset $D \subset \partial K(D$ may consists of a single point) there exists a supporting hyperplane of $K$ containing $D$ (an $m-1$-dimensional hyperplane $E$ is a supporting hyperplane of $K$ if $E \subset E_{m}$ and $E \cap K=E \cap \partial K \neq \emptyset$, a supporting half-space of $K$ in $E_{m}$ is a closed half-space with $E$ as its boundary and that contains $K$ );

(iv) $K \subset E_{m}$ is the intersection of all its supporting half-spaces.

(v) $P \in K$ is called an extreme point of $K$ if $P$ cannot be represented as a convex combination of other points in $K . P$ is called an exposed point of $K$ if there exists an supporting hyperplane $E_{P}$ of $K$ such that $E_{P} \cap K=\{P\}$. We denote by the set of all extreme points of $K$ by $K_{e}$.

Notice that for every compact set $W \subset \mathbb{R}^{n},[C(W)]_{e} \subset W$. Therefore we write $[C(W)]_{e}$ as $W_{\rho}$.

Definition 2.9. (the supporting chain). - Suppose $K \subset M^{N \times n}$ is compact and convex. Suppose the dimension of $K$ is $m_{1} \geq 1$ and $K$ is contained in a plane $E_{1} \subset M^{N \times n}$ with the same dimension.

If $E_{1}$ does not have rank-one connections, or $K$ consists of only one element, we define $\mathcal{E}=\left\{E_{m_{1}}\right\}$.

If $E_{1}$ has rank-one connections and $E_{1} \cap K$ has more than one element, let $\mathcal{E}=\left\{E_{i}, i=1, \ldots, k\right\}$ be a collection of planes with dimensions $m_{1}$, $0 \leq m_{k}<m_{k-1}<\ldots<m_{1}$, such that

(i) $E_{j+1} \subset E_{j}$, for $j=1, \ldots k-1$,

(ii) $E_{i}$ has rank-one connections for $i=1, \ldots, k-1$ and $E_{i} \cap K$ has more than one element, while $E_{k}$ does not have rank-one connections or $E_{k} \cap K$ consists of only one element.

(iii) for each $i<k, E_{i}^{\prime}$ is a supporting plane of $E_{i} \cap K$ with dimension $m n_{i} \cdots 1$, such that $E_{i}^{\prime} \cap K$ is of dimension $m_{i+1}$ and $E_{i}^{\prime} \cap K \subset E_{i+1}$ so that $E_{i}^{\prime} \cap K=E_{i+1} \cap K$. 
Then we call $\mathcal{E}=\left\{E_{1}, \cdots, E_{k}\right\}$ a supporting chains of $K$, and call $E_{k}$ the end plane. For simplicity we denote the end plane $E_{k}$ of $\mathcal{E}$ by $E_{\varepsilon}$.

It will be shown in Theorem 1.2 below that every extreme point of $K$ must be contained in one of the supporting chains of $K$. It is easy to see that if $E_{\mathcal{E}}$ consists of only one point, that point must be an extreme point of $K$.

The following is a result for quasiconvex hulls of sets contained in a plane without rank-one connections. It is a consequence of [BFJK, Th. 4.1]:

Proposition 2.10. - Suppose $K \subset E \subset M^{N \times n}$, where $K$ is a closed set and $E$ is a plane without rank-one connections. Then

$$
Q_{2}(K)=K
$$

Since in this proposition, we do not assume boundedness of $K$, we use $Q_{2}(K)$. If $K$ is bounded, $Q(K)=K$.

Proof. - Notice that quasiconvex hulls are translation invariant, that is, if we define $K+P=\{A+P, A \in K\}$ for a fixed $P \in M^{N \times n}$, then $Q_{p}(K+P)=Q_{p}(K)+P$ for all $p \in[1, \infty)$. Therefore, without loss of generality, we may assume that $E$ is a subspace of $M^{N \times n}$ without rank-one matrices. It was established in [BFJK] that there exists a constant : > 0 , such that

$$
\int_{\Omega}\left|P_{E^{\perp}}(D \phi)\right|^{2} d x \geq e \int_{\Omega}|D \phi|^{2} d x
$$

for every open set $\Omega \subset \mathbb{R}^{n}$ and every $\phi \in C_{0}^{\infty}\left(\Omega, \mathbb{R}^{N}\right)$.

Suppose $P \in Q_{2}(K)$, we see that $P \in E$ and we have, from Proposition 2.2 that there exists a sequence $\phi_{j} \in C_{0}^{\infty}\left(D, \mathbb{R}^{N}\right)$ such that

$$
0=\operatorname{dist}^{2}(P, K)=\lim _{j \rightarrow \infty} \int_{D} \operatorname{dist}^{2}\left(P+D \phi_{j}(x), K\right) d x
$$

$D$ being the unit hypercube in $\mathbb{R}^{n}$. Since $K \subset E$, we then have, from the fact

$$
\operatorname{dist}^{2}(A, K) \geq\left|P_{E^{\perp}}(A)\right|^{2}
$$

that $\int_{D}\left|P_{E^{\perp}}\left(D \phi_{j}(x)\right)\right|^{2} d x \rightarrow 0 \quad$ as $\quad j \rightarrow \infty$. Therefore, $\lim _{j \rightarrow \infty} \int_{D}$ $\left|D \phi_{j}(x)\right|^{2} d x=0$. Hence $\operatorname{dist}^{2}(P, K)=0$ which implies $P \in K$. 


\section{PROOFS OF MAIN RESULTS}

We need the following lemma for the proofs of our results.

Lemma 3.1. - Suppose $K \subset M^{N \times n}$ is compact and let $P \in Q(K)$. Then there exists a HYM $\nu$ supported in $K$ such that $\bar{\nu}_{K}=P$. In particular, when $P \in Q(K) \backslash K, \nu \neq \delta_{P}$.

Proof. - For every fixed $P \in Q(K)$, we have, from Proposition 2.5, $Q \operatorname{dist}(P, K)=0$. Then Proposition 2.2 implies that there exists a sequence $\left(\phi_{j}\right)$ in $C_{0}^{\infty}\left(D, \mathbb{R}^{N}\right)$ such that

$$
\lim _{j \rightarrow \infty} \int_{D} \operatorname{dist}\left(P+D \phi_{j}(x), K\right) d x=0
$$

where $D \subset \mathbb{R}^{n}$ is the unit cube $D=(0,1)^{n}$. Since $K$ is bounded, $D \phi_{j}$ is equi-integrable in $D$. Therefore, from Dunford-Pettis theorem (see [ET]), up to a subsequence, $\phi_{j} \rightarrow \phi$ in $W_{0}^{1,1}\left(D, \mathbb{R}^{N}\right)$ weakly as $j \rightarrow \infty$. We extend $\phi_{j}$ periodically to $\mathbb{R}^{n}$ and then define

$$
z_{j}(x)=\frac{1}{j} \phi_{j}(j x)
$$

It is casy to see that $z_{j} \rightarrow 0$ in $W_{0}^{1,1}\left(D, \mathbb{R}^{N}\right)$ weakly, and

$$
\lim _{j \rightarrow \infty} \int_{D} \operatorname{dist}\left(P+D z_{j}(x), K\right) d x=0 .
$$

Applying a result in $[\mathrm{Zl}]$, up to a subsequence, we may have a bounded sequence $\left(g_{j}\right)$ in $W^{1, \infty}\left(D, \mathbb{R}^{N}\right)$ such that,

$$
\lim _{j \rightarrow \infty} \int_{D}\left|D z_{j}-D g_{j}\right| d x=0
$$

so that

$$
\lim _{j \rightarrow \infty} \int_{D} \operatorname{dist}\left(P+D g_{j}(x), K\right) d x=0 .
$$

Let $\left\{\nu_{x}\right\}_{x \in D}$ be the family of gradient Young measures corresponding to $\left(P+D g_{j}\right)$. It is easy to see that $\operatorname{supp} \nu_{x} \subset K$ and $\int_{K} \lambda d \nu_{x}=P$ for almost every $x \in D$. We then have, from Lemma 2.7 that there exists a HYM $\nu$, such that $\operatorname{supp} \nu \subset K$ and $\bar{\nu}_{K}=P$. The second claim is a direct consequence of the fact $\bar{\nu}_{K}=P$. So, $\nu=\delta_{P}$ implies $P \in K$. 
Proof of Theorem 1.1. - We prove (i) by contradiction. Suppose $K \backslash Q\left(K_{q, c}\right) \neq \emptyset$, we have, from Definitions 2.3, 2.4 and Proposition 2.5 that $Q \operatorname{dist}\left(P, Q\left(K_{q, f^{*}}\right)\right)>0$ whenever $P \notin Q\left(K_{q, e}\right)$. Let

$$
\alpha=\max \left\{Q \operatorname{dist}\left(P, Q\left(K_{q, e}\right)\right), P \in K\right\}
$$

We have $\alpha>0$. Let

$$
K_{1}=\left\{P \in K, Q \operatorname{dist}\left(P, Q\left(K_{q, e}\right)\right)=\alpha\right\},
$$

then $K_{1} \subset K$ is nonempty and compact. From the definition of quasiconvex hulls, we see that $Q\left(K_{1}\right) \subset K$. Let $P_{0}$ be a quasiconvex extreme point of $K_{1}$. It is easy to see that $P_{0} \in K_{1}$. We seek to prove that $P_{0} \in K_{q, e}$ to reach a contradiction. Let $\nu$ be a HYM supported in $K$ such that $\bar{\nu}_{K}=P_{0}$. Since $Q \operatorname{dist}\left(\cdot, Q\left(K_{q, e}\right)\right)$ is quasiconvex, (2.4) implies

$$
\int_{K} Q \operatorname{dist}\left(\lambda, Q\left(K_{q, e}\right)\right) d \nu \geq Q \operatorname{dist}\left(P_{0}, Q\left(K_{q, e}\right)\right)=\alpha .
$$

On the other hand,

$$
\begin{gathered}
\int_{K} Q \operatorname{dist}\left(\lambda, Q\left(K_{q, e}\right)\right) d \nu \\
=\int_{K_{1}} Q \operatorname{dist}\left(\lambda, Q\left(K_{q, e}\right)\right) d \nu+\int_{K \backslash\left[Q\left(K_{q, e}\right) \cup K_{1}\right]} Q \operatorname{dist}\left(\lambda, Q\left(K_{q, e}\right)\right) d \nu \\
=I_{1}+I_{2} \\
I_{1}=\int_{K_{i}} Q \operatorname{dist}\left(\lambda, Q\left(K_{q, e}\right)\right) d \nu=\alpha \nu\left(K_{1}\right) .
\end{gathered}
$$

Since $\nu(K)=1$, we see that

$$
I_{2}=\int_{K \backslash\left(Q\left(K_{q, c} \cup K_{1}\right)\right.} Q \operatorname{dist}\left(\lambda, Q\left(K_{q, e}\right)\right) d \nu=0,
$$

which gives $\nu\left(K \backslash K_{1}\right)=0$. Otherwise, $I_{2}<\alpha \nu\left(K \backslash K_{1}\right)$ so that

$$
\alpha<\alpha \nu\left(K_{1}\right)+\alpha \nu\left(K \backslash K_{1}\right)=\alpha
$$

Contradiction. Therefore $\nu\left(K_{1}\right)=1$ and hence supp $\nu \subset K_{1}$. Since $P_{0}$ is a quasiconvex extreme point of $K_{1}, \nu=\delta_{P_{0}}$. This contradicts to the fact that $P_{0} \notin K_{q, e}$. The proof of (i) is complete. 
Now we prove (ii). Since $Q(W)=K$, let $P \in K_{q, e} \subset K$, we have, from Lemma 3.1, there exists a HYM $\nu$ supported in $W$, such that $\bar{\nu}_{W}=P$. Since $W \subset K$, we conclude from the definition of $K_{q, e}$ that $\nu=\delta_{P}$. Hence $P \in W$.

In the proof of Theorem 1.1, we used the fact that $Q \operatorname{dist}\left(P, Q\left(K_{q, e}\right)\right)>0$ whenever $P \notin Q\left(K_{q, e}\right)$. In fact, a stronger result was established in [Z3] that for all $K \subset M^{N \times n}$ and all $p \in[1, \infty)$,

$$
Q \operatorname{dist}^{p}(\cdot K)=Q \operatorname{dist}^{p}\left(\cdot, Q_{p}(K)\right) .
$$

Proof of Theorem 1.2. - Let $P_{0} \in K_{e}$ and supp $\subset \subset K$, such that $\bar{\nu}_{K}=P_{0}$. We use a dimension reduction argument and try to find a supporting chain with the end plane containing $P_{0}$. Suppose the dimension of $C(K)$ is $m_{1}$ and $C(K)$ is contained in a $m_{1}$-dimensional plane $E_{1}$, $1 \leq m_{1} \leq N n$ (if $m_{1}=0$ ), the set $K$ consists of only one point, and the claim is then trivially true). Let $E_{1}^{\prime}$ be a supporting hyperplane of $C(K)$ in $E_{1}$ containing $P_{0}$. Since $C(K)$ is on one side of the supporting hyperplane $E_{m_{1}-1}, \operatorname{supp} \nu \subset K \subset C(K), \int_{K} \lambda d \nu=P_{0}$, and $\nu$ is a probability measure, we see that $\operatorname{supp} \nu \subset K \cap E_{1}^{\prime}$. Notice that $C(K) \cap E_{1}^{\prime}=C\left(K \cap E_{1}^{\prime}\right)$. Let the dimension of $C\left(K \cap E_{1}^{\prime}\right)$ be $m_{2}$ and let $C\left(K \cap E_{1}^{\prime}\right)$ be contained in an $m_{2}$-dimensional plane $E_{2}$. If $E_{2}$ does not have rank-one connections, or $m_{2}=0$, we have, from [BFJK, Th. 4.1] that $\nu=\delta_{P_{0}}$. If $E_{2}$ has rank-one connections, notice that $P_{0}$ is also an extreme point of $C\left(K \cap E_{2}\right)$. Repeat the previous step, we have a finite number of planes $E_{k} \supset E_{k-1} \supset \cdots \supset E_{1}$, with $0 \leq m_{k}<m_{k-1}<\cdots<m_{1} \leq n N$, such that $\operatorname{supp} \nu \subset E_{k} \cap K$. We see that either $E_{k}$ does not have rank-one connections, so that we conclude the proof, or the dimension of $C\left(K \cap E_{k}\right)$ is zero so that $E_{k} \cap K=\left\{P_{0}\right\}$ and the conclusion follows, that is, $\operatorname{supp} \nu=\delta_{P_{0}}$.

As pointed out to the author by the referee, there is a much simpler proof of Theorem 1.2 by using measure-thereotical description of extreme points (see [A]). $P \in K$ is an extreme point of $C(K)$ if and only if for any probability measure $\nu$ supported in $C(K)$, such that $\nu_{C(K)}=P$ implies $\nu=\delta_{P}$. Since the set of gradient homogeneous Young measures is a subclass of all probability measures, the conclusion follows. However, the proof of Theorem 1.2 by using the supporting chain $\mathcal{E}=\left\{E_{1}, \cdots, E_{k}\right\}$ with the end plane $E_{k}$ containing $P_{0}$ is more suitable for the study of quasiconvex extreme points as we will see again in the proof of Theorem 1.5.

Proof of Theorem 1.3. - We prove this results by three steps.

$$
\operatorname{STEP}(1) .-[Q(K)]_{q, e} \subset K \text {. }
$$


Since $K \subset Q(K)$, we need only to show that for every $P \in Q(K) \backslash K$, $P \notin[Q(K)]_{q, e}$. This follows from Lemma 3.1 that there exists a HYM $\nu$ such that $\operatorname{supp} \nu \subset K$ and $\bar{\nu}_{K}=P$. Since $P \notin K, \nu \neq \delta_{P}$. Step (1) is proved.

STEP (2). $-[Q(K)]_{q, e} \subset K_{q, e}$.

Suppose $P \in[Q(K)]_{q, \mathrm{e}}$. We see from Step (1) that $P \in K$. For any HYM $\nu$, such that supp $\nu \subset K$ and $\bar{\nu}_{K}=P$, we notice that $K \subset Q(K)$. By the definition of $[Q(K)]_{4, \epsilon}$, we have $\nu=\delta_{P}$, hence $P \in K_{4, \cdots}$

STEP (3). $-K_{q, e} \subset \overline{\left[Q(K)_{q, e}\right]}$.

Let $P \in K_{q, e}$. From Theorem 1.1 and Lemma 3.1, we see that there exists a homogeneous Young measure $\nu, \operatorname{supp} \nu \subset \overline{\left[Q(K)_{q, e}\right]}$ (notice that $Q(K)_{q, e}$ is not necessarily closed) such that $\int_{\left[Q(K)_{q, e}\right]} \lambda d \nu(\lambda)=P$. Since $\overline{\left[Q(K)_{q, e}\right]} \subset K$, we see that $\nu=\delta_{P}$. Hence $P \in \overline{\left[\left(Q(K)_{q, e}\right]\right.}$. The proof is complete.

Theorem 1.1 is crucial for the proof of Step (3) of Theorem 1.3, because it provides a representation of $P$ in $Q(K)$ by the closure of its smallest generator $\overline{\left[Q(K)_{l, e}\right]}$, that is,

$$
P=\int_{\overline{\left[Q(K)_{4, r]}\right]}} \lambda d \nu(\lambda)
$$

However, Theorem 1.1 does not provide any further information on the HYM $\nu$. It can happen that for some $P \in Q(K)$, there are many different HYM supported in $\overline{\left[Q(K)_{q, e}\right]}$ with integral average $P$. We can easily find examples of compact convex sets in $M^{1 \times n} \cong \mathbb{R}^{n}$ such that this happens.

Proof of Theorem 1.4. - Proposition 2.10 implies that $K=Q(K)$. Let $P \in K$ and let $\nu$ be a HYM supported in $K$ such that $\bar{\nu}_{K}=P$. [BFJK, Th. 4.1] implies that $\nu=\delta_{P}$.

Proof of Theorem 1.5. - The idea of proof is similar to that of Theorem 1.2 , though it is more complicated. Let $m_{1} \geq 1$ be the dimension of $K$. There exists an $m_{1}$-dimensional plane $E_{1}$ which contains $K$ and which has rank-one connections. We prove this result in three steps.

STEP (1). - $\quad K_{q, e} \subset \partial K$, where $\partial K$ is the relative boundary of $K$ in $E_{1}$.

Since for every $P \in \operatorname{int} K$, there exists a rank-one matrix $R \in M^{N \times n}$ and $t_{1}>0, t_{2}>0$, such that $P+t_{1} R \in K, P-t_{2} R \in K . P$ is a convex combination of these two matrices. A classical result on two-well problem in $[\mathrm{BJ} 1]$ by a direct lamination construction shows that there exists 
HYM $\nu$ supported in $K_{0}:=\left\{P+t_{1} R, P-t_{2} R\right\}$ such that $\bar{\nu}_{K_{0}}=P$. Hence $P \notin K_{q, e}$.

$$
\text { STEP (2). - } \quad K_{q, e} \subset \bigcup_{\mathcal{E}}\left(E_{\mathcal{E}} \cap K\right) \text {. }
$$

Let $P \in K_{q, e}$. From Step (1), we see that $P \in \partial K$. There is a supporting hyperplane $E_{1}^{\prime}$ in $E_{1}, P \in K \cap E_{1}^{\prime}$. Suppose the dimension of $K \cap E_{1}^{\prime}$ is $m_{2}$ and $K \cap E_{1}^{\prime}$ is contained in a plane $E_{2}$.

If $E_{2}$ does not have rank-one connections or consists of only $P$, we see that $K \cap E_{1}^{\prime} \subset K_{q, e}$ and $P \in E_{\mathcal{E}}$ with $\mathcal{E}=\left\{E_{1}, E_{2}\right\}$. The proof of Step (2) is then finished.

If $E_{2}$ has rank-one connections, we see from Step (1) that $P \in \partial\left(K \cap E_{2}\right)$ - the relative boundary in $E_{2}$. Then we repeat the previous argument. We obtain a supporting chain $\mathcal{E}=\left\{E_{1}, E_{2}, \ldots, E_{k}\right\}$ with the dimensions of the planes satisfying $N n \geq m_{1}>m_{2}>\cdots>m_{k} \geq 0$. We have $P \in K \cap E_{k}$, and $E_{k}$ either consists of only one matrix $P$ or does not have rank-one connections. In both cases, $P \in \bigcup_{\mathcal{E}}\left(E_{\mathcal{E}} \cap K\right)$.

STEP (3). - $\quad \bigcup_{\mathcal{E}}\left(E_{\mathcal{E}} \cap K\right) \subset K_{q, \mathcal{E}}$.

Let $P \in \bigcup_{\mathcal{C}}\left(E_{\mathcal{E}} \cap K\right)$. There exists a supporting chain

$$
\mathcal{E}=\left\{E_{1}, \cdots, E_{k}=E_{\mathcal{E}}\right\}
$$

such that

$$
P \in E_{\mathcal{E}} \cap K \subset E_{k-1}^{\prime} \cap K \subset \cdots \subset E_{1} \cap K=K
$$

Let $\nu$ be a HYM supported in $K, \bar{\nu}_{K}=P$. If $k=1, \mathcal{E}=\left\{E_{1}\right\}$. Then $E_{1}$ does not have rank-one connections, $K \subset E_{1}$. Theorem 1.4 implies that $K=K_{q, e}=K \cap E_{1}$, hence $\nu=\delta_{P}, P \in K_{q, e}$. If $k>1$, we have supp $/ \subset K \cap E_{1}^{\prime}$ because $E_{1}^{\prime}$ is a supporting plane of $K$ passing through $P$. Since $K \cap E_{1}^{\prime}=K \cap E_{2}$. Repeating this we see that $\operatorname{supp} \nu \subset K \cap E_{k}$, while either $E_{k}$ does not have rank-one connections or $K \cap E_{k}=\{P\}$. In both cases, we have $\nu=\delta_{P}$, thus $P \in K_{q, e}$.

\section{EXAMPLES}

Example 4.1. - When $K \subset M^{1, n} \cong \mathbb{R}^{n}$, the gradient Young measures are generated by sequences of gradients of scalar-valued functions. In this case $K_{q, e}=K_{e}$ because for all $A, B \in M^{1 \times n}, A \neq B$, we have $\operatorname{rank}(A-B)=1$. Therefore we can embed all examples of compact 
convex sets from [Ro] into this case. In particular, there exists a compact convex set in $\mathbb{R}^{3}$, such that $K_{\mathrm{r}}$ is not closed [Ro, page 167]. Therefore there exists $K \subset M^{1 \times 3}$ such that $K_{q, E}$ is not closed. We consider this as a trivial example of non-compactness of $K_{\text {q.e. }}$.

Recall Theorem 1.4 that another extreme case of $K_{q, e}$ is when $K$ is contained in a plane $E \subset M^{N \times n}$ without rank-one connections. In this case for every compact subset $K \subset E, K_{q, e}=K=Q(K)$. Yet another example is $K=S O(n) H$, the so-called one-well structure. We have $K_{\text {q. } e^{\prime}}=K=Q(K)$ (see for example [Re, K, BJ1, BJ2, BFJK]).

Example 4.2. - We show that there exists a compact set $K \subset M^{2 \times 2}$ such that $Q(K) \neq C(K), K_{q, r} \neq K_{r}$ and $K_{q, *}$ is not closed.

Let

$$
\begin{aligned}
K=\left\{A(t, s) \in M^{2 \times 2}\right. & \\
A(t, s) & \left.=\left(\begin{array}{cc}
s & t \\
-t & 0
\end{array}\right),-1 \leq s \leq 1,0 \leq t \leq 1, t s=0\right\} .
\end{aligned}
$$

Obviously, $K_{\epsilon}=\left\{A_{+}, A_{-}, A_{0}\right\}$, where

$$
A_{+}=\left(\begin{array}{ll}
1 & 0 \\
0 & 0
\end{array}\right) . \quad A_{-}=\left(\begin{array}{cc}
-1 & 0 \\
0 & 0
\end{array}\right), \quad A_{0}=\left(\begin{array}{cc}
0 & 1 \\
-1 & 0
\end{array}\right) .
$$

We show that

(i) $K_{q, \epsilon}=\left\{A_{+}, A_{-}, t A_{0}, 0<t \leq 1\right\}$.

It is easy to see that $A_{+}, A_{-}, A_{0} \in K_{q, \epsilon}$. Notice that $\operatorname{rank}\left(A_{+}-A_{-}\right)=1$. Following from Theorem 1.2, we see that

$$
\left\{s A_{+}+(1-s) A_{-}, 0<s<1\right\} \cap K_{4, r}=\emptyset .
$$

We need to prove that $t A_{0} \in K_{q, e}$ for $0<t<1$. Let $P=t A_{0}$ and let $\nu$ be a HYM supported in $K$, such that $\bar{\nu}_{K}=P$. We use a method in [Sv2] to show that an approximate sequence is a Cauchy sequence. Notice that for any $A\left(t_{1}, s_{1}\right), A\left(t_{2}, s_{2}\right) \in K$ with $-1 \leq s_{1}, s_{2} \leq 1,0 \leq t_{1}, t_{2} \leq 1, s_{1} t_{1}=0, s_{2} t_{2}=0$,

$$
\operatorname{det}\left[A\left(t_{1}, s_{1}\right)-A\left(t_{2}, s_{2}\right)\right]=\left(t_{1}-t_{2}\right)^{2} .
$$

Let $\left(P+D \phi_{j}\right)$ be a generating sequence of $\nu$ such that $\phi_{j}$ converges to 0 in $W_{0}^{1, \infty}\left(D, \mathbb{R}^{2}\right)$ in the weak-* sense, where $D$ is the unit square in $\mathbb{R}^{2}$. Since

$$
\lim _{j \rightarrow \infty} \int_{D} \operatorname{dist}\left(P+D \phi_{j}, K\right) d x \rightarrow 0,
$$


we see that for any $A \in \operatorname{supp} \nu$, we have $a_{21}=-a_{12}$ where $a_{21}, a_{21}$ being the $(1,2)$ and $(2,1)$ entries of matrix $A$. We can also see, for any $\epsilon>0$, that

$$
\lim _{j \rightarrow 0} \operatorname{meas}\left(\left\{x \in D, \operatorname{dist}\left(P+D \phi_{j}(x), K\right) \geq \epsilon\right\}\right)=0 .
$$

Let

$$
I_{i, j}=\int_{D} \operatorname{det}\left[\left(P+D \phi_{j}(x)\right)-\left(P+D \phi_{i}(x)\right)\right] d x .
$$

On one hand, we see from the weak-* continuity of Jacobians [Bl1], that

$$
\lim _{j \rightarrow \infty} \lim _{i \rightarrow \infty} I_{i, j}=0
$$

On the other hand, from (4.1) and (4.2), we have

$$
\lim _{j \rightarrow \infty} \lim _{i \rightarrow \infty} I_{i, j}=\int_{K}\left[\int_{K^{i}}\left(\lambda_{12}-\tau_{12}\right)^{2} d \nu(\lambda)\right] d \nu(\tau)=0 .
$$

Let $\phi_{j}=\left(\phi_{j}^{(1)}, \phi_{j}^{(2)}\right)$. A similar argument to that in [Sv2] gives

$$
\frac{\partial \phi_{j}^{(1)}}{\partial x_{2}} \rightarrow 0, \quad \frac{\partial \phi_{j}^{(2)}}{\partial x_{1}} \rightarrow 0 \quad \frac{\partial \phi_{j}^{(2)}}{\partial x_{2}} \rightarrow 0,
$$

almost everywhere in $D$ up to a subsequence. From the fact that

$$
\lim _{j \rightarrow 0} \operatorname{meas}\left(\left\{x \in D, \operatorname{dist}\left(P+D \phi_{j}(x), K\right) \geq \epsilon\right\}\right)=0,
$$

and the special structure of $K$, we can also conclude that

$$
\frac{\partial \phi_{j}^{(1)}}{\partial x_{1}} \rightarrow 0
$$

almost everywhere. Hence $\nu=\delta_{P}, P \in K_{q, \epsilon}, K_{q, t}$ is not closed.

(ii) $K=Q(K)$.

Let $P \in Q(K)$. Since $Q(K) \subset C(K)$, we may assume that

$$
P=\left(\begin{array}{cc}
a & b \\
-b & 0
\end{array}\right)
$$

with $0<b<1$. From Lemma 3.1, we see that there exists a HYM $\nu$ supported in $K$, such that $P=\bar{\nu}_{K}$. We can use a similar argument as in (i) to show that $a=0$, hence $P \in K$. The proof is left to the reader. 
Example 4.3. - We show that there exists $K \subset M^{2 \times 2}$ such that $Q(K) \neq C(K), K_{q, \cdot}=K_{\text {. }}$

Let

$$
S_{+}=\left\{A \in M^{2 \times 2} ; A=\left(\begin{array}{ll}
a & c \\
c & b
\end{array}\right), a \geq 0, b \geq 0, \operatorname{det} A=0\right\} .
$$

$S_{+}$can be parameterized as

$$
S_{+}=\left\{A \in M^{2 \times 2} ; A=\left(\begin{array}{cc}
\sqrt{x^{2}+y^{2}}+x & y \\
y & \sqrt{x^{2}+y^{2}}-x
\end{array}\right), x, y \in \mathbb{R}\right\} .
$$

Let

$$
L=\left\{A \in M^{2 \times 2} ; A=\left(\begin{array}{cc}
1+x & y \\
y & 1-x
\end{array}\right), x, y \in \mathbb{R}\right\}
$$

be a plane in the subspace of all $2 \times 2$ symmetric matrices. Define $K$ be the set of symmetric matrices in $S_{+}$such that they are on the same side of $L$ as the zero matrix. We can prove that

(i) $S_{+} \cap L$ is a circle. $K_{t^{\prime}}$ consists of the zero matrix and $S_{+} \cap L$. This is easy to see.

(ii) $K_{q, \text { : }}=K_{\epsilon}$. To see this, we have, for every $P \notin K_{\epsilon}$, there exists some $t>1$ such that $t P \in S_{+} \cap L$. Since $t P$ is a rank-one matrix, $P$ is a convex combination of the zero matrix and $t P$, we see that $P \notin K_{q, \epsilon}$. Hence $K_{q, c} \subset K_{e}$. Theorem 1.2 implies $K_{q, \epsilon} \supset K_{\epsilon}$. The conclusion follows.

(iii) $K=Q(K)$. Suppose $P \in Q(K)$, we have $P \in C(K)$ and there exists a HYM $\nu$ supported in $K_{q, e}$ (notice here that $K_{q, e}$ is closed) such that $P=\bar{\nu}_{h_{q, .}}$. Since Jacobians are weakly continuous ([B11]), we see that

$$
\int_{K_{q, \sigma}^{-}} \operatorname{det} \lambda d \nu(\lambda)=\operatorname{det} \bar{\nu}_{K_{q, \varepsilon}}=\operatorname{det} P \text {. }
$$

Since $\operatorname{det} \lambda=0$ for $\lambda \in K$, we see that $\operatorname{det} P=0$, hence $P \in K$. The proof is finished.

Example 4.4. - Suppose $f: M^{N \times n} \rightarrow[0,+\infty)$ is quasiconvex, satisfying $f^{-1}(0) \neq \emptyset$ and

$$
K_{\alpha}=\left\{P \in M^{N \times n}, f(P) \leq \alpha\right\}
$$

is compact for some $\alpha>0$. We see that $K_{\alpha}$ is quasiconvex and that $K_{\alpha}$ contains some interior points of $M^{N \times n}$. We may claim, following the same 
argument of proof of Theorem 1.2 or that of Theorem 1.5, Step (1), that $\left(K_{u}\right)_{q, e}$ is contained in $\partial K_{c x}$ which is a subset of $\left\{P \in M^{N \times n}, f(P)-\alpha\right\}$. Therefore, for every $P \in K_{\alpha}$, there exists a HYM $\nu$ supported in $\partial K_{\alpha}$ such that

$$
P=\bar{\nu}_{\partial K_{r}}=\int_{\partial K_{n}} \lambda d \nu(\lambda)
$$

\section{APPLICATIONS TO TWO-WELL AND THREE-WELL PROBLEMS}

In this section we use results established in previous sections and some properties of special quasiconvex functions to find quasiconvex extreme points for the sets $K_{2}=S O(n) \cup S O(n) H$ and $K_{3}=\cup_{k=1}^{3}\left[S O(n) H_{k}\right]$, where $H$ and $H_{k}$ are distinct positive definite matrices in $M^{n \times n}$, and $S O(n)$ is the set of all orthogonal matrices in $M^{n \times n}$ with determinant 1 , $n>1, S O(n) H=\{R H, R \in S O(n)\}$.

The quasiconvex hull $Q(S O(n) \cup S O(n) H)$ is not known except for a few special cases [Ma, Sv2, MS].

Let $I_{n}$ be the identity matrix in $M^{n \times n}$. We have

THEOREM 5.1. - Let $H \neq I_{n}$ be positive definite. Then for $K_{2}=$ $S O(n) \cup S O(n) H,\left(K_{2}\right)_{q, e}=K_{2}$.

Remark 5.1. - A direct consequence of Theorem 5.1 is that $S O(n) \cup$ $S O(n) H$ is the smallest closed generator of $Q(S O(n) \cup S O(n) H)$.

The following example shows that a similar statement is not true in general for three or more wells.

Example 5.1. - Consider $S O(n), S O(n) I_{\lambda}, S O(n) I_{\tau}$, where

$$
I_{\lambda}=\left(\begin{array}{cc}
I_{n-1} & 0 \\
0 & \lambda
\end{array}\right), \quad I_{\tau}=\left(\begin{array}{cc}
I_{n-1} & 0 \\
0 & \tau
\end{array}\right)
$$

with $1<\lambda<\tau$.

Let $K_{3}=S O(n) \cup S O(n) I_{\lambda} \cup S O(n) I_{\tau}$. It is easy to see that for every $R \in S O(n), R I_{\lambda}$ is a convex combination of $R \in S O(n)$ and $R I_{\tau}$, and $\operatorname{rank}\left(R-R I_{\tau}\right)=1$. Hence $R I_{\lambda} \notin\left(K_{3}\right)_{q, e}$ so that $\left[S O(n) I_{\lambda}\right] \cap\left(K_{3}\right)_{q, e}=\emptyset$.

The following result provides some information for $\left(K_{3}\right)_{q, e}$, where $K_{3}=\cup_{k=1}^{3}\left[S O(n) H_{k}\right]$. 
THEOREM 5.2. $-\left(K_{3}\right)_{q, e}$ equals either $K_{3}$ or $S O(n) H_{k} \cup S O(n) H_{j}$ for some fixed $k \neq j, 1 \leq k, j \leq 3$.

This result implies that if for some $P \in S O(n) H_{k}$ and some $k$, $P \in\left(K_{3}\right)_{q, e}$, then the whole set $S O(n) H_{k}$ is contained in $\left(K_{3}\right)_{q, e}$, and $\left(K_{3}\right)_{q, e}$ is always compact.

Proof of Theorem 5.1. - It is well known now that

$$
\begin{gathered}
Q(S O(n))=S O(n)=[S O(n)]_{q, e}, \\
Q(S O(n) H)=S O(n) H=[S O(n) H]_{q, e}
\end{gathered}
$$

(see, for example [Re, K, Bl3]). From Proposition 2.2 and Definition 2.4, we see that

$$
f(P)=Q \operatorname{dist}^{2}(P, S O(n)) \quad \text { and } \quad f_{H}(P)=Q \operatorname{dist}^{2}(P, S O(n) H)
$$

are quasiconvex, nonnegative and vanish on $S O(n)$ and $S O(n) H$ respectively. Another property of $f$ and $f_{H}$ is that they are rotation invariant in the sense that

$$
f(R P)=f(P), \quad f_{H}(R P)=f_{H}(P)
$$

for every fixed $P \in M^{n \times n}$ and all $R \in S O(n)$. Therefore, there are two constants $\alpha_{1}>0, \alpha_{2}>0$, such that $f(P)=\alpha_{1}>0$ for all $P \in S O(n) H$, and $f_{H}(P)=\alpha_{2}>0$ for all $P \in S O(n)$.

Now, for $P \in S O(n) H$, let $\nu$ be a HYM, supp $\nu \subset K_{2}$, such that $\bar{\nu}_{K_{2}}=P$. Since $f(\cdot)$ is quasiconvex, we have, on one hand,

$$
\int_{K_{2}} f(\lambda) d \nu(\lambda) \geq f\left(\bar{\nu}_{K_{2}}\right)=f(P)=\alpha_{1}
$$

On the other hand, $\alpha_{1}-\max \left\{f(A), A \in K_{2}\right\}$. Therefore

$$
\operatorname{supp} \nu \subset\left\{A \in K_{2}, f(A)=\alpha_{1}\right\}=S O(n) H .
$$

Hence $\nu=\delta_{P}$. This shows that $S O(n) H \subset\left(K_{2}\right)_{q, e}$. The case when $P \in S O(n)$ is similar if we use $f_{H}$ instead.

Proof of Theorem 5.2. - We will prove that

(i) if $S O(n) H_{i} \cap Q\left(S O(n) H_{j} \cup S O(n) H_{k}\right)=\emptyset$, for all $i \neq j \neq k$, $1 \leq i, j, k \leq 3$, then $\left(K_{3}\right)_{q, e}=K_{3}$; 
(ii) if for some $i, j, k$,

$$
S O(n) H_{i} \cap Q\left(S O(n) H_{j} \cup S O(n) H_{k}\right) \neq \emptyset .
$$

Then $\left(K_{3}\right)_{q, e}=S O(n) H_{j} \cup S O(n) H_{k}$.

For case (i), notice that $f_{i}(P)=\operatorname{dist}^{2}\left(P, S O(n) H_{j} \cup S O(n) H_{k}\right)$ is rotation invariant for every fixed $P \in M^{n \times n}$ in the sense that $f(R P)=f(P)$ for all $R \in S O(n)$. Then it is not hard to see by applying Proposition 2.2 that $Q f_{i}$ is also rotation invariant. Now, if we follow the same argument for $P \in S O(n) H_{i}$ by using $Q f_{i}$ as in the proof of Theorem 5.1 , we see that $S O(n) H_{i} \subset\left(K_{3}\right)_{q, e}$. Hence $\left(K_{3}\right)_{q, e}=K_{3}$.

For case (ii), without loss of generality, we may assume that $i=1$, $j=2, k=3$. We prove this claim by three simple steps.

STEP (1)

$$
\left(K_{3}\right)_{q, e} \subset S O(n) H_{2} \cup S O(n) H_{3} .
$$

In fact, we prove that $S O(n) H_{1} \subset Q\left(S O(n) H_{2} \cup S O(n) H_{3}\right)$ so that every point in $S O(n) H_{1}$ can be represented by nontrivial HYMs supported in $S O(n) H_{2} \cup S O(n) H_{3}$ by Lemma 3.1. Therefore $S O(n) H_{1}$ does not contain any quasiconvex extreme points of $K_{3}$, that is, $S O(n) H_{1} \cap\left(K_{3}\right)_{q, e}=\emptyset$. Hence the conclusion of Step (1) will follow.

By a similar argument to that in case (i), we see that

$$
F_{1}(P)=Q \operatorname{dist}^{2}\left(P,\left[S O(n) H_{2} \cup S O(n) H_{3}\right]\right)
$$

is quasiconvex, rotation invariant and vanishs on $Q\left(S O(n) H_{1} \cup S O(n) H_{3}\right)$. From the assumption, for some $P_{0} \in S O(n) H_{1}, F_{1}\left(P_{0}\right)=0$. Since $F_{1}$ is rotation invariant, we see that $F_{1}(P)=0$ for all $P \in S O(n) H_{1}$, hence

$$
S O(n) H_{1} \subset Q\left(S O(n) H_{2} \cup S O(n) H_{3}\right) \text {. }
$$

so that $S O(n) H_{1} \cap\left(K_{3}\right)_{q, e}=\emptyset$.

STEP (2)

$$
\begin{aligned}
& S O(n) H_{2} \cap Q\left(S O(n) H_{1} \cup S O(n) H_{3}\right)=\emptyset, \\
& S O(n) H_{3} \cap Q\left(S O(n) H_{1} \cup S O(n) H_{2}\right)=\emptyset .
\end{aligned}
$$

We prove the first equality only. If the claim were not true, that is,

$$
S O(n) H_{2} \cap Q\left(S O(n) H_{1} \cup S O(n) H_{3}\right) \neq \emptyset \text {, }
$$


a similar argument to that of Step (1) would give

$$
S O(n) H_{2} \subset Q\left(S O(n) H_{1} \cup S O(n) H_{3}\right) .
$$

so that $S O(n) H_{2} \cap\left(K_{3}\right)_{a, c^{\prime}}=\emptyset$. Since $\left(K_{3}\right)_{q, e} \subset K_{3}$, we see that $\left(K_{3}\right)_{q, e} \subset S O(n) H_{3}$. However, it is known that $Q\left(S O(n) H_{3}\right)=S O(n) H_{3}$ which does not contain $K_{3}$. Contradiction.

STEP (3). $-\left(K_{3}\right)_{q, e}=S O(n) H_{2} \cup S O(n) H_{3}$.

Notice that

$$
\begin{aligned}
& F_{2}(P)=Q \operatorname{dist}^{2}\left(P, S O(n) H_{1} \cup S O(n) H_{3}\right), \\
& F_{3}(P)-Q \operatorname{dist}^{2}\left(P, S O(n) H_{1} \cup S O(n) H_{2}\right)
\end{aligned}
$$

are quasiconvex, rotation invariant, $F_{2}(P)=\alpha_{2}>0$ for $P \in S O(n) H_{2}$, $F_{3}(P)=\alpha_{3}>0$ for $P \in S O(n) H_{3}$, where $\alpha_{2}, \alpha_{3}$ are constants. Just as in the proof of Theorem 5.1, we may conclude that $S O(n) H_{2} \subset\left(K_{3}\right)_{q, r}$, $S O(n) H_{3} \subset\left(K_{3}\right)_{q, f}$. Combining these with the conclusion of Step (1), we see that

$$
\left(K_{3}\right)_{l, e}=S O(n) H_{2} \cup S O(n) H_{3} .
$$

Remark 5.1. - For the multi-well case, we may obtain similar results as those of Theorem 5.2. Let $K_{m}=\cup_{i=1}^{m} S O(n) H_{i}$, where $H_{i}$ 's are distinct positive definite $n \times n$ matrices and $m \geq 3$. We see that $\left(K_{m}\right)_{q, e}=\cup_{j=1}^{k} S O(n) H_{i,}$ for some $k \geq 2(k \leq m)$. An interesting observation from this is that $\left(K_{m}\right)_{q, r}$ either contains the whole well $S O(n) H_{i}$ or does not intersect with it.

\section{ACKNOWLEDGEMENT}

I would like to thank John. M. Ball, Jan Kristensen, Zamin Iqbal and the referee for helpful suggestions.

\section{APPENDIX}

Proof of Proposition 2.5. - (see [Z2]) Let $K_{1}=\left\{X \in M^{N \times n}\right.$, $\left.Q \operatorname{dist}^{p}(X, K)=0\right\}$. Obviously, $Q^{(s)}(K) \subset K_{1}$. Let $f: M^{N \times n} \rightarrow \mathbb{R}$ be any quasiconvex function. Let

$$
\alpha_{f}=\sup _{X \in K} f(X)
$$


and

$$
f_{\alpha_{f}}(X)=\max \left\{f(X)-\alpha_{f}, 0\right\}
$$

It is easy to see that $f_{\alpha_{f}}$ is quasiconvex, $Q K \subset f_{\alpha_{f}}^{-1}(0)$ and $Q^{(*)}(K)=$ $\cap_{f} f_{\alpha_{f}}^{-1}(0)$. We may assume that $f_{\alpha_{f}}^{-1}(0)$ is compact, otherwise take the convex function

$$
g(\cdot)=\operatorname{dist}^{2}(\cdot, C(K))
$$

which is the squared distance function to a convex set. Therefore $f_{\alpha_{f}}+g$ is quasiconvex. We claim that $\left(f_{\alpha_{f}}+g\right)^{-1}(0) \subset C(K)$, hence it is compact. This is easy to see because $f_{\alpha_{f}} \geq 0$ and $g^{-1}(0)=C(K)$. We have, for any fixed $1 \leq p<\infty$,

$$
Q \operatorname{dist}^{p}\left(X, f_{\alpha_{f}}^{-1}(0)\right) \leq \operatorname{dist}^{p}\left(X, f_{\alpha_{f}}^{-1}(0)\right) \leq \operatorname{dist}^{p}(X, K)
$$

for all $X \in M^{N \times n}$. Since $Q \operatorname{dist}^{p}\left(X, f_{\alpha_{f}}^{-1}(0)\right)$ is quasiconvex, we have

$$
Q \operatorname{dist}^{p}\left(X, f_{\alpha_{j}}^{-1}(0)\right) \leq Q \operatorname{dist}^{p}(X, K)
$$

From [Z1, Th. 1.1] and its proof, we see that for a compact zero set $f_{\alpha_{f}}^{-1}(0)$ corresponding to a nonnegative quasiconvex function $f_{\alpha_{f}}$, and for any $1 \leq p<\infty$,

$$
f_{\alpha_{f}}^{-1}(0)=\left\{X \in M^{N \times n},{Q \operatorname{dist}^{p}}^{p}\left(X, f_{\alpha_{f}}^{-1}(0)\right)=0\right\} .
$$

Hence, $K_{1} \subset f_{\alpha_{f}}^{-1}(0)$ for every quasiconvex function $f$, thus $K_{1} \subset Q^{(s)}(K)$. The proof is complete.

\section{REFERENCES}

[A] E. M. Alfsen, Compact Convex Sets and Boundary Integrals. Springer-Verlag, 1971.

[AF] E. ACERBI and N. FusCo, Semicontinuity problems in the calculus of variations. Arch. Rational Mech. Anal., Vol. 86, 1984, pp. 125-145.

[BL] H. BERLIOCCHI, J. M. LASRY, Intégrandes normales et mesures paramétrées en calcul des variations. Bull. Soc. Math. France, Vol. 101, 1973, pp. 129-184.

[BH] J. M. BALL, Convexity conditions and existence theorems in nonlinear elasticity. Arch. Rational Mech. Anal., Vol. 63, 1977, pp. 337-403.

[BI2] J. M. BALL, A version of the fundamental theorem of Young measures, in Partial Differential Equations and Continuum Models of Phase Transitions, (edited by $\mathrm{M}$. Rascle, D. Serre and M. Slemrod), 1989, pp. 207-215, Springer-Verlag.

[B13] J. M. BalL, Sets of gradients with no rank-one connections. J. Math. Pures et Appl., Vol. 69, 1990, pp. 241-259. 
[BFJK] K. Bhattacharya. N. B. Firoozye, R. D. James, R. V. Kohn, Restrictions on Microstructures. Proc. Royal Soc. Edinbargh, A, Vol. 124, 1994, pp. 843-878.

[BJI] J. M. BALL, R. D. James, Fine phase nixlures as minimizers of energy. Arch. Rational Mech. Anal, Vol. 100, 1987. pp. 13-52.

$\lceil\mathrm{BJ} 2\rceil$ J. M. BAL.L, R. D. JAMES. Proposed experimental tests of a theory of fine microstructures and the two-well problem. Phil. Royal Soc: Lon, Vol. 338A, 1992, pp. 389-450.

[BZ] J. M. BALL and K.-W. ZHANG, Lower semicontinuity and multiple integrals and the biting lemma . Proc. Royal Soc. Edinburgh, Vol. 114A, 1990, pp. 367-379.

[CK] M. СHIPOT, D. KindERLEHRER, Equilibrium configurations of crystals Arh. Rational Mech. Anal, Vol. 103, 1988, pp. 237-277.

[D] B. Dacorogna, Direct Methods in the Calculus of Variations, Springer-Verlag, 1989.

[ET] I. Ekeland, R. Temam, Convex Aanlysis and Variational Problems, North-Holland. 1976.

[K] D. KinderLeHrER, Remarks about equilibrium configurations of crystals, in Material Instabilities in Continuum Mechanics, J. M. BAl.L. ed., Oxford University Press, 1988, pp. 977-83.

[KP] D. Kinderlehrer, P. Pedregal, Characterizations of Young measures generated by gradients. Arch. Rational Mech. Anal, Vol. 115, 1991, pp. 329-365.

[Ma] J. P. MATOS, Young measures and the absence of fine microstructures in a class of phase transitions. European J. Appl. Math, Vol. 3, 1992, pp. 31-54.

[Mo] C. B. Ji Morrey, Multiple integrals in the calculus of variations, Springer, 1966.

[MS] S. MÜlLER, V. Šverák, Attainment results for the two-well problem by convex integration, preprint, 1993.

[Rel Yu. G. Reshernak, Liouville's theorem on conformal mappings under minimal regularity assumptions. Siberian Math. J., Vol. 8, 1967, pp. 631-653.

[Ro] R. T. Rockafellar, Convex Analysis, Princeton University Press, 1970.

[Ru] W. Rudin, Functional Analysis, McGraw-Hill, 1973.

[Sv1] V. S̆VERAK, On the problem of two wells, preprint.

[Sv2] V. S̆verák, On Tartar's conjecture. Ann. Inst. H. Poincaré, Vol. 10, 1993, pp. 405-412.

[Sv3] V. S̆verák, Rank one convexity does not imply quasiconvexity. Proc. Royal Soc. Edin., Vol. 120A, 1992, pp. 185-189.

[T] L TARTAR, Compensated compactness and applications to partial differential equations, in Nonlinear Analysis and Mechanics: Heriot-Watt Symposium, IV, R. J. Knops ed Pitman, 1979.

[Y] B.-S. YAN, Remarks on the set of quasi-conformal matrices in higher dimensions, Preprint, 1994.

[Z1] K.-W. ZHANG, A construction of quasiconvex functions with linear growth at infinity. Ann. Sc. Nom. Sup. Pisa Serie IV, Vol. XIX, 1992, pp. 313-326.

[Z2] K.-W. Zhang, On non-negative quasiconvex functions with unbounded zero sets, Proc. Royal Soc. Edin. Vol. 127A, 1997, pp. 411-422.

[Z3] K.-W. ZHANG, On some quasiconvex functions with linear growth, to appear in J. Convex Anal.

(Manuscript received June 17, 1996;

Revised version received September 19, 1996.) 